\title{
Closing an on-going clinical trial: when is it betrayal of participants?
}

\author{
Cynthia Chauhan · Mary Lou Smith
}

Received: 10 October 2013/ Accepted: 12 October 2013/Published online: 26 October 2013

(C) The Author(s) 2013. This article is published with open access at Springerlink.com

We have become aware of several instances of precipitous and, in our view, egregious and unjustified closures of ongoing clinical trials in which a substantial number of patients were already participating in investigational efforts, some involving biopsies for research purposes. These closures raise serious ethical issues for the research community. We will discuss those issues and some possible changes in how trials are conducted to address the problem. It is our premise that closing on-going clinical trials without scientific, efficacy, or safety justification is an abhorrent affront to all participants in clinical research as well as a fundamental betrayal of the trust that motivates patient participants to enroll in clinical trials.

Cancer patients who accept the risk of an investigational drug are true partners in bringing new agents to market. They hope they will benefit but, regardless of personal benefit/response, they hope the researchers will learn something to help other patients. Patients participate in clinical research for multiple reasons but, particularly in the case of agreeing to undergo mandatory research biopsies, do so because the research has the potential to improve the care, treatment approach, and standards for cancer patients. They engage in a relationship with researchers based on their trust in the integrity of the researchers and the system within which the researchers work. Any cavalier approach to the commitment patients make to research is indefensible and particularly reprehensible when participants undergo internal organ biopsies. Violating the trust of these patients also violates the trust the patients place in the investigators, undermining patient confidence in and availability for research. That trust and

C. Chauhan $(\square) \cdot$ M. L. Smith

31 N. Laurel Drive, Wichita, KS 67206, USA

e-mail: cynthiachauhan@aol.com any violation of it are deepened when the researcher is also a given patient's treating oncologist.

It is with good reason that human beings who enroll in clinical trials are called participants, not subjects. A participant is one who takes part in something-an active, volitional partner or colleague. A subject is one, mouse or human, who is under the power or authority and at the incontestable will of another or others. That difference between a participant and a subject is significant and germane to this discussion of when and why it is or is not appropriate to close a clinical trial. Human participants have a choice about joining a trial and that choice is heavily influenced by the participants' perceptions of the integrity, ethics, and trustworthiness of the investigators and sponsors.

The travesty of sponsors unilaterally closing on-going clinical trials for business reasons not related to the science, efficacy, or safety of the trial may be the shared secret of the cancer research community. Some are silent because they are perpetrators. Some are silent because they are fearful of loss or retribution for speaking. Most tragically, some, patient participants, are victims unaware. Whatever the reasons, the lack of active outrage is deeply troubling. We, the advocates and researchers, must ask ourselves are we looking the other way when we should be standing up for patients and challenging the process? Is our silence simply acceptance, or worse, apathy? Consider Martin Niemoeller's reported observation on his own silence: "...they came for ....[others] so I did not speak out. And when they came for me, there was no one left to speak out for me."

Unilateral trial closing absent safety concerns, unplanned analyses of the outcome of the study, or changes in the risk/benefits threatens the foundation of clinical research and betrays trust. Precipitous and inappropriate closure of 
an on-going clinical trial has far-reaching implications that raise significant issues about the ethics of clinical trials and the trust that patients exhibit by participating in the clinical research process. Some of the potential implications include the participants being treated as lab rats, reinforcing an old, negative stereotype of clinical trials; reduction of enrollment in future trials; and diminishment of the reputation of both the researchers and the research sites as well as the sponsors. These are issues that need to be seriously considered.

Any early termination decision, especially in trials where patients agree to biopsies for research purposes only, must have scientific, efficacy, or safety justification. Closure for any other reason, such as realigning financial investments or changing priorities, is a blatant violation of the trust patients place when signing a consent form. Early trial closures not related to the conduct of the trial negatively affect the science that can be learned from the biopsies collected due to the much smaller sample size. The ability to make conclusions on the correlative studies becomes underpowered, substantially compromising the risk-benefit of performing these procedures.

Well-conducted, focused research has the best potential for advancing the treatment and possible cure of cancer patients; therefore, it is imperative that scientific decisions be based on scientific criteria. Those scientific decisions must be the basis of trial continuation or closure. Therefore, we recommend that Phase II studies of a certain size or complexity have an independent Data and Safety Monitoring Board.

Closing clinical studies for non-scientific, non-safety, or non-efficacy reasons is not only unacceptable and unconscionable but also violates the moral and ethical standards of beneficent research with human participants. The appropriate action is to complete the study as specified in the protocol. It is in the best interest of the patients and the science to do so. The larger issue is that measures must be in place to prevent actions of this kind in the future, as failure to do so will undermine translational research with human participants, an essential link in the drive to reduce and, someday, remove the burden of cancer.

Those of us who are involved in the conduct of cancer research, advocates, researchers, and sponsors, must be aware that research involving human participants is a contract with participants, not a use of subjects. Therefore, we must ask ourselves this question: If failure to fully honor the research contract with cancer patients does not bother us, what will?

Conflict of interest The authors declare that they have no conflict of interest.

Open Access This article is distributed under the terms of the Creative Commons Attribution Noncommercial License which permits any noncommercial use, distribution, and reproduction in any medium, provided the original author(s) and the source are credited. 